\title{
Diagnósticos de enfermagem mais utilizados em casos confirmados de covid-19 no
}

\section{Brasil}

\author{
Most used nursing diagnoses in confirmed cases of covid-19 in Brazil \\ Diagnósticos de enfermería más utilizados en casos confirmados de covid-19 en Brasil
}

Recebido: 06/06/2021 | Revisado: 15/06/2021 | Aceito: 21/06/2021 | Publicado: 04/07/2021

Thays Vieira Cusato

ORCID: https://orcid.org/0000-0002-3766-6530

Associação Brasileira de Enfermeiros Cientistas, Brasil

E-mail: thays.vieira.cusato@gmail.com

Judith Victoria Castillo Mejía

ORCID: https://orcid.org/0000-0001-5257-6878

Associação Brasileira de Enfermeiros Cientistas, Brasil

E-mail: iker07castillo@live.com

Priscila Gramata da Silva Vitorino

ORCID: https://orcid.org/0000-0002-1201-6945

Associação Brasileira de Enfermeiros Cientistas, Brasil

E-mail: prigramaenf@yahoo.com.br

Daiana Moreira Gomes

ORCID: https://orcid.org/0000-0001-9387-0619

Associação Brasileira de Enfermeiros Cientistas, Brasil E-mail: daigomes_87@hotmail.com

Daniele Vignoli Ribeiro

ORCID: https://orcid.org/0000-0001-5296-8302

Associação Brasileira de Enfermeiros Cientistas, Brasil E-mail: vignoliribeiro@gmail.com

Luana de Oliveira Hernandes

ORCID: https://orcid.org/0000-0001-8828-7146 Associação Brasileira de Enfermeiros Cientistas, Brasil

E-mail: luanaoliveira2306@gmail.com

Thais Oliveira de Paula Lima

ORCID: https://orcid.org/0000-0002-0706-1098

Associação Brasileira de Enfermeiros Cientistas, Brasil E-mail: thaisoplima@gmail.com

Natasha Vila Chã

ORCID: https://orcid.org/0000-0002-9781-454X Associação Brasileira de Enfermeiros Cientistas, Brasil E-mail: natashavilacha@ hotmail.com

Victor Hugo de Paula Flauzino

ORCID: https://orcid.org/0000-0001-5156-0030 Associação Brasileira de Enfermeiros Cientistas, Brasil

E-mail: prof.victorflauzino@unyleya.edu.br

Jonas Magno dos Santos Cesário

ORCID: https://orcid.org/0000-0003-1785-3555

Associação Brasileira de Enfermeiros Cientistas, Brasil

E-mail: prof.jonasmagno@unyleya.edu.br

\section{Resumo}

No momento em que o paciente evolui para o quadro grave da covid-19, o enfermeiro deve sistematizar, planejar e direcionar à assistência de enfermagem para que ocorra a recuperação deste paciente. Deste modo, este estudo possui o objetivo central de identificar os diagnósticos de enfermagem mais frequentes em pacientes contaminados pela covid-19. O estudo foi realizado por meio de uma revisão bibliográfica de abordagem descritiva e qualitativa, em bibliotecas digitais, que permitem uma busca em bases de dados próprias por meio da utilização de descritores padronizados. Foi possível identificar 12 diagnósticos frequentes em pacientes com covid-19, que são: promoção da saúde: proteção ineficaz, risco de desequilíbrio eletrolítico, diarreia, débito cardíaco diminuído, sofrimento espiritual, hipertermia relacionada, dor aguda relacionada. Por fim, constatou-se uma escassez de publicações nacionais a respeito do tema abordado nesta pesquisa, com isto os autores esperam que este estudo contribua para o desenvolvimento de novas pesquisas relacionadas aos diagnósticos de enfermagem em pacientes acometidos pelo covid-19. Quando bem aplicado, o DE impacta de maneira positiva na assistência de enfermagem e contribuir para uma melhor recuperação do paciente, além de evitar intervenções desnecessárias.

Palavras-chave: Infecções por coronavírus; Diagnóstico de enfermagem; Plano de assistência de enfermagem. 


\begin{abstract}
When the patient progresses to the severe condition of covid-19, the nurse must systematize, plan and direct nursing care so that the patient's recovery occurs. Thus, this study has the central objective of identifying the most frequent nursing diagnoses in patients contaminated by covid-19. The study was carried out through a literature review with a descriptive and qualitative approach, in digital libraries, which allow a search in their own databases using standardized descriptors. It was possible to identify 12 frequent diagnoses in patients with covid-19, which are: health promotion: ineffective protection, risk of electrolyte imbalance, diarrhea, decreased cardiac output, spiritual distress, related hyperthermia, related acute pain. Finally, there was a shortage of national publications on the topic addressed in this research, so authors expect this study to contribute to the development of new research related to nursing diagnoses in patients affected by covid-19. When properly applied, the ND has a positive impact on nursing care and contributes to a better patient's recovery, in addition to avoiding unnecessary interventions.
\end{abstract}

Keywords: Coronavirus infections; Nursing diagnosis; Nursing careplan.

\title{
Resumen
}

Cuando el paciente progresa a la condición severa de covid-19, la enfermera debe sistematizar, planificar y dirigir los cuidados de enfermería para que se produzca la recuperación del paciente. Así, este estudio tiene como objetivo central identificar los diagnósticos de enfermería más frecuentes en pacientes contaminados por covid-19. El estudio se llevó a cabo mediante una revisión de la literatura con un enfoque descriptivo y cualitativo, en bibliotecas digitales, que permiten una búsqueda en sus propias bases de datos mediante el uso de descriptores estandarizados. Fue posible identificar 12 diagnósticos frecuentes en pacientes con covid-19, los cuales son: promoción de la salud: protección ineficaz, riesgo de desequilibrio electrolítico, diarrea, disminución del gasto cardíaco, angustia espiritual, hipertermia relacionada, dolor agudo relacionado. Finalmente, hubo una falta de publicaciones nacionales sobre el tema abordado en esta investigación, por lo que los autores esperan que este estudio contribuya al desarrollo de nuevas investigaciones relacionadas con los diagnósticos de enfermería en pacientes afectados por covid-19. Cuando se aplica correctamente, el ND tiene un impacto positivo en los cuidados de enfermería y contribuye a una mejor recuperación del paciente, además de evitar intervenciones innecesarias.

Palabras clave: Infecciones por coronavirus; Diagnóstico de enfermería; Plan de atención a enfermería.

\section{Introdução}

Em dezembro de 2019, na cidade de Wuhan, na China, surgiram os primeiros casos de pneumonia viral com causa desconhecida e altamente transmissível. Em janeiro de 2020, os chineses isolaram um novo coronavírus, o qual denominaram de Sars-cov2, que já afetava muitos países de vários continentes. Em fevereiro, a doença foi nomeada oficialmente como covid-19 pela Organização Mundial da Saúde (OMS), em 11 de março de 2020 foi decretada a pandemia mundial (Cesário et al., 2020c \& Dantas et al., 2020).

Em 2020, o covid-19 foi responsável por infectar milhões de pessoas e se espalhar por todo o mundo rapidamente. Após 3 meses de ter sido decretado a pandemia mundial, já existiam 6 milhões de casos registrados e em dezembro daquele ano esse número ultrapassou os 70 milhões. Em junho de 2021, este número ultrapassou a marca dos 170 milhões de indivíduos infectados e milhares de mortes já haviam sido registradas. O Brasil enfrentou um rápido avanço da doença, com mais de 16 milhões de casos e aproximadamente 500 mil óbitos. (John Hopkins Coronavirus Resource Center, 2021).

De acordo com Dantas et al. (2020), os pacientes infectados pela covid-19 podem apresentar sintomas leves, similares a uma síndrome gripal, que pode agravar e desenvolver a síndrome da angústia respiratória aguda (SARA) (Gallaschi et al., 2020). No momento em que o paciente evolui para o quadro grave da covid-19, o enfermeiro deve sistematizar, planejar e direcionar à assistência de enfermagem para que ocorra a recuperação deste paciente. (Cesário et al. 2020b).

Medeiros (2020) descreve a Sistematização da Assistência de Enfermagem (SAE) como uma linguagem padronizada, utilizada pela equipe de enfermagem para direcionar todo o cuidado ofertado ao paciente. A SAE é importante para padronizar toda a rotina da equipe e um de seus principais instrumentos metodológicos é o processo de enfermagem (PE), constituído por 5 (cinco) etapas: histórico de enfermagem, diagnóstico de enfermagem, planejamento, implementação e avaliação de enfermagem (Cesário et al. 2020b \& Humerez, Ohl e Silva, 2020).

O diagnóstico de enfermagem (DE) é uma ação privativa do enfermeiro e consiste em um julgamento clínico das necessidades humanas básicas afetadas ou das respostas humanas apresentadas pelo paciente, família ou comunidade, para que 
seja elaborado um plano de cuidados individualizado para direcionar as intervenções de enfermagem e oferecer um cuidado holístico (Queiroz et al., 2020 \& Sousa et al., 2020).

Neste contexto, a pandemia da covid-19 trouxe um novo desafio global para a enfermagem, que precisou se adaptar rapidamente para atender essa nova demanda. Ao considerar que o enfermeiro utiliza a SAE como instrumento norteador para atender os pacientes, o que inclui aqueles infectados pelo novo coronavírus, levantou-se o um questionamento sobre quais os diagnósticos de enfermagem mais utilizados em pacientes acometidos pela covid-19. Deste modo, este estudo possui o objetivo central de identificar os diagnósticos de enfermagem mais frequentes em pacientes contaminados pela covid-19.

\section{Metodologia}

O estudo foi realizado por meio de uma revisão bibliográfica de abordagem descritiva e qualitativa, descrita por Cesário, Flauzino e Mejia (2020) como um tipo de pesquisa baseada em material já construído, como artigos científicos publicados em periódicos acadêmicos. Primeiramente buscou-se reunir evidências para responder à pergunta de pesquisa $\mathrm{e}$ para essa etapa inicial, a busca foi realizada em bibliotecas digitais, que permitem uma busca em bases de dados próprias por meio da utilização de descritores padronizados.

No DeCS (Descritores em Ciências da Saúde) foram encontrados os seguintes descritores: infecções por coronavírus, diagnóstico de enfermagem e plano de assistência de enfermagem. As bibliotecas bancos de dados utilizados foram o Google Acadêmico, BVS (biblioteca virtual em saúde) e SciELO (Scientific Electronic Library Online). No Google Acadêmico utilizou-se cada um dos descritores entre aspas ("“"). Na BVS foi utilizado a opção pesquisa avançada, selecionadas as bases da BDENF (Banco de Dados em Enfermagem), LILACS (Literatura Latino-Americana e do Caribe em Ciências da Saúde) e o operador lógico booleano "OR" e "AND". Na SciELO, foi utilizada a opção pesquisa avançada e o operador lógico booleano "OR" e "AND".

Estabeleceu-se como critérios de inclusão, artigos acadêmicos publicados entre 2020 e 2021, na língua portuguesa, disponíveis de forma gratuita e nos bancos de dados já mencionados, que respondessem à pergunta de pesquisa. Excluíram-se artigos repetidos encontrados nas bases de dados, resumos, artigos inferiores a 2020 e artigos que não respondiam o problema da pesquisa. A coleta dos dados foi realizada no mês de março, por 3 pesquisadores de forma independente.

Os resultados das buscas pelos dados e do número final de publicações que compuseram a revisão são apresentados conforme orientações do prisma na forma de fluxograma, conforme Figura 1 (Peters, 2015). 
Figura 1. Diagrama de fluxo dos artigos encontrados.

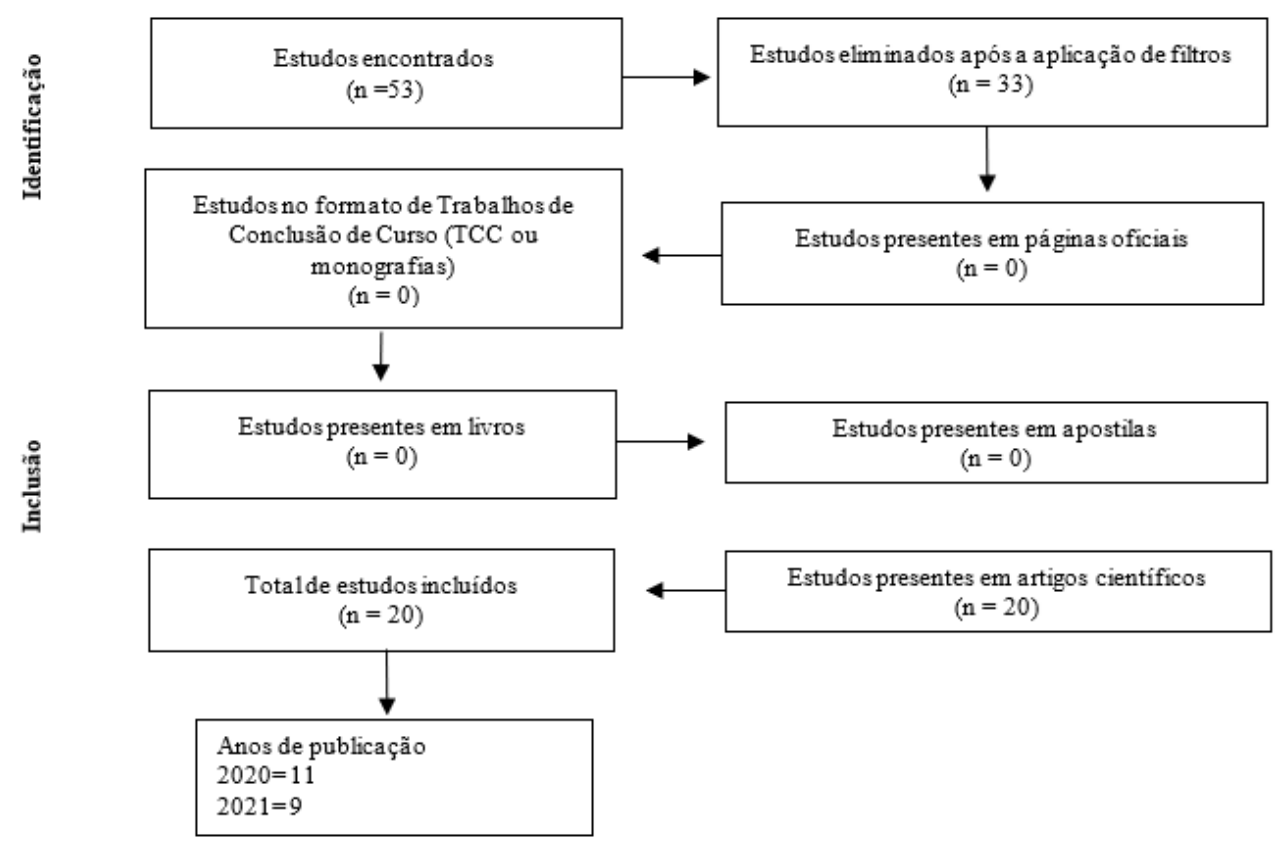

Fonte: elaborado pelos autores (2021).

\section{Resultados}

Para uma melhor organização dos artigos foi desenvolvida a Tabela 1, que mostra a distribuição inicial dos artigos científicos encontrados nas bases de dados da BVS, ScIELO e Google Acadêmico. É possível notar que o maior aproveitamento de periódicos foi na base do Google Acadêmico com 14 artigos incluídos, na BVS, 5 periódicos foram inclusos e na ScIELO, somente 1 artigo foi aproveitado, o que resultou em uma amostra final de 20 artigos científicos.

Tabela 1. Resultados da busca nas bases de dados

\begin{tabular}{c|c|c}
\multirow{2}{*}{ Base de Dados } & \multicolumn{2}{|c}{ Artigos } \\
\cline { 2 - 3 } & Total & Incluídos \\
\hline BVS & 20 & 5 \\
\hline Google Acadêmico & 24 & 14 \\
\hline ScIELO & 9 & 1 \\
\hline Total & $\mathbf{5 3}$ & $\mathbf{2 0}$ \\
\hline
\end{tabular}

Fonte: Elaborado pelos autores (2021)

O Quadro 1 foi desenvolvido para apresentar um melhor entendimento dos periódicos selecionados nas bases de dados, pois permite a comparação entre os assuntos abordados em cada um dos estudos selecionados. Este quadro foi confeccionado com as seguintes variáveis: autor, título, objetivo central e tipo de estudo. No Quadro 1 é possível notar a relação dos artigos científicos com os objetivos da pesquisa. 
Quadro 1. Artigos inclusos neste estudo.

\begin{tabular}{|c|c|c|c|}
\hline Autor/ano & Título & Objetivos & Tipo de estudo \\
\hline Andrade et al., 2020 & $\begin{array}{l}\text { Principais diagnósticos de } \\
\text { enfermagem em pacientes com } \\
\text { manifestações clínicas da covid-19 }\end{array}$ & $\begin{array}{l}\text { Relacionar os aspectos clínicos e a } \\
\text { sintomatologia dos pacientes com os } \\
\text { diagnósticos de enfermagem aplicados no } \\
\text { contexto da covid-19 através da taxonomia da } \\
\text { Associação Norte-Americana de Diagnósticos } \\
\text { de Enfermagem Internacional (NANDA-I) } \\
\text { versão 2018-2020. }\end{array}$ & $\begin{array}{l}\text { Pesquisa exploratória e } \\
\text { bibliográfica }\end{array}$ \\
\hline Andrade et al., 2021 & $\begin{array}{l}\text { Assistência de enfermagem aos } \\
\text { casos leves da covid-19 }\end{array}$ & $\begin{array}{l}\text { Identificar os possíveis diagnósticos e } \\
\text { intervenções de enfermagem em pacientes } \\
\text { acometidos pela forma leve da covid-19 } \\
\text { utilizando a taxonomia da Classificação } \\
\text { Internacional para a prática de enfermagem } \\
\text { (CIPE) versão 2019. }\end{array}$ & $\begin{array}{l}\text { Revisão integrativa da } \\
\text { literatura }\end{array}$ \\
\hline Cardoso et al., 2020 & $\begin{array}{l}\text { Diagnóstico, resultados e } \\
\text { intervenções de enfermagem ao } \\
\text { idoso frágil, acometido por covid- } \\
\text { 19: uma análise documental }\end{array}$ & $\begin{array}{l}\text { Elencar os principais diagnósticos, resultados e } \\
\text { intervenções de enfermagem ao idoso frágil na } \\
\text { Instituição de longa permanência para idosos } \\
\text { (ILPI) e no domicílio acometido por covid-19. }\end{array}$ & $\begin{array}{l}\text { Revisão documental, } \\
\text { com abordagem } \\
\text { qualitativa, descritiva e } \\
\text { exploratório }\end{array}$ \\
\hline Cesário et al., 2021 & $\begin{array}{l}\text { Cuidados de enfermagem durante a } \\
\text { anestesia de paciente com covid-19 }\end{array}$ & $\begin{array}{l}\text { Descrever os principais cuidados de } \\
\text { enfermagem e as suas principais intercorrências } \\
\text { durante anestesia de paciente com covid-19 }\end{array}$ & $\begin{array}{l}\text { Revisão bibliográfica de } \\
\text { abordagem descritiva e } \\
\text { qualitativa }\end{array}$ \\
\hline Dias et al., 2020 & $\begin{array}{l}\text { Diagnósticos de enfermagem para } \\
\text { pacientes com síndrome } \\
\text { inflamatória multissistêmica } \\
\text { pediátrica associada à covid-19: } \\
\text { revisão integrativa }\end{array}$ & $\begin{array}{l}\text { Identificar afirmativas diagnósticas de } \\
\text { enfermagem para pacientes com Síndrome } \\
\text { Inflamatória Multissistêmica Pediátrica (SIM- } \\
\text { P) associada à covid-19, a partir dos } \\
\text { indicadores evidenciados na literatura } \\
\text { cientifica. }\end{array}$ & Revisão integrativa \\
\hline Dias et al., 2021 & $\begin{array}{l}\text { Diagnósticos e intervenções de } \\
\text { enfermagem a crianças com sinais } \\
\text { respiratórios de gravidade da covid- } \\
19\end{array}$ & $\begin{array}{l}\text { Descrever afirmativas de diagnósticos e de } \\
\text { intervenções de enfermagem a crianças com } \\
\text { sinais respiratórios de gravidade da covid-19 }\end{array}$ & $\begin{array}{l}\text { Estudo exploratório, } \\
\text { descritivo, do tipo } \\
\text { revisão narrativa }\end{array}$ \\
\hline Melo et al., 2021 & $\begin{array}{l}\text { Processo de enfermagem aplicado } \\
\text { ao paciente com covid-19 a partir } \\
\text { de uma scoping review }\end{array}$ & $\begin{array}{l}\text { Propor o processo de enfermagem para } \\
\text { pacientes com infecção pela covid-19 a partir } \\
\text { de evidências disponíveis acerca das } \\
\text { características clínicas }\end{array}$ & $\begin{array}{l}\text { Scoping review de } \\
\text { caráter descritivo, com } \\
\text { abordagem quantitativa }\end{array}$ \\
\hline Menezes et al., 2020 & $\begin{array}{l}\text { Terminologia especializada de } \\
\text { enfermagem para a prática clínica à } \\
\text { covid-19 }\end{array}$ & $\begin{array}{l}\text { Construir uma terminologia especializada para } \\
\text { a prática clínica de enfermagem a pessoas com } \\
\text { covid-19 fundamentada no Modelo de Sete }\end{array}$ & $\begin{array}{lr}\text { Estudo } & \text { descritivo, } \\
\text { documental } & \text { orientado } \\
\text { pela primeira e segunda }\end{array}$ \\
\hline
\end{tabular}




\begin{tabular}{|c|c|c|c|}
\hline & & $\begin{array}{l}\text { Eixos da Classificação Internacional para a } \\
\text { prática de Enfermagem }\end{array}$ & $\begin{array}{l}\text { etapas das diretrizes de } \\
\text { elaboração } \\
\text { subconjuntos } \\
\text { terminológicos, que são } \\
\text { a identificação dos } \\
\text { termos e o mapeamento } \\
\text { entre os termos } \\
\text { identificados e a CIPE®, } \\
\text { respectivamente }\end{array}$ \\
\hline Moura et al., 2020 & $\begin{array}{l}\text { NANDA covid-19": relato da } \\
\text { construção de panfleto educativo } \\
\text { sobre os diagnósticos de } \\
\text { enfermagem ás pessoas infectadas } \\
\text { com o SARS-CoV-2" }\end{array}$ & $\begin{array}{l}\text { Relatar a construção de panfleto educativo } \\
\text { sobre os diagnósticos de enfermagem ás } \\
\text { pessoas infectadas com o SARS-CoV-2 }\end{array}$ & 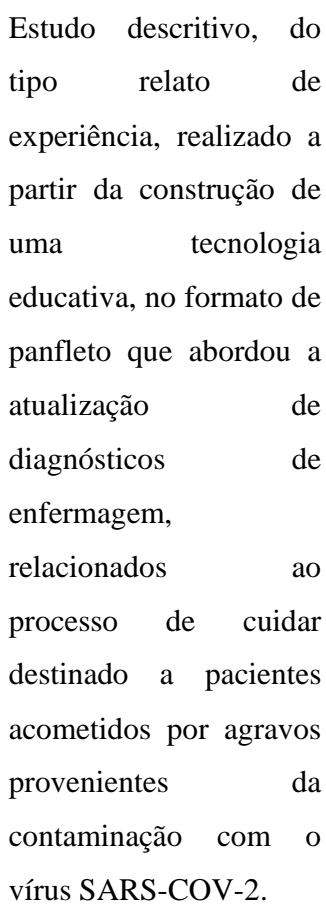 \\
\hline $\begin{array}{l}\text { Nascimento et al., } \\
2021\end{array}$ & \begin{tabular}{llr} 
Infecções por & \multicolumn{2}{c}{ coronavírus: } \\
planejamento da & \multicolumn{2}{c}{ assistência } \\
fundamentado na & Teoria de \\
Enfermagem de Orem & &
\end{tabular} & $\begin{array}{l}\text { Relatar a experiência de docentes e discentes de } \\
\text { uma disciplina de pós-graduação sobre } \\
\text { assistência de enfermagem no combate ao novo } \\
\text { coronavírus (covid-19) fundamentada na Teoria } \\
\text { do Autocuidado }\end{array}$ & $\begin{array}{l}\text { Revisão bibliográfica da } \\
\text { literatura } \\
\text { apresentações } \\
\text { seminários }\end{array}$ \\
\hline Queiroz et al., 2020 & $\begin{array}{l}\text { Diagnósticos de enfermagem } \\
\text { segundo a taxonomia da NANDA } \\
\text { internacional para sistematização da } \\
\text { assistência de enfermagem a covid- } \\
19 .\end{array}$ & $\begin{array}{l}\text { Narrar as manifestações clínicas das vítimas da } \\
\text { covid-19 com base em evidências científicas e } \\
\text { propor os principais diagnósticos de } \\
\text { enfermagem de acordo com a Taxonomia da } \\
\text { NANDA Internacional. }\end{array}$ & $\begin{array}{l}\text { Estudo exploratório } \\
\text { qualitativo, descritivo } \\
\text { realizado por meio de } \\
\text { revisão bibliográfica. }\end{array}$ \\
\hline Ramos et al., 2020 & $\begin{array}{l}\text { Diagnósticos, resultados e } \\
\text { intervenções de enfermagem } \\
\text { voltados para comunidade, na } \\
\text { pandemia de covid-19 }\end{array}$ & $\begin{array}{l}\text { Identificar os diagnósticos de enfermagem } \\
\text { segundo a taxonomia da NANDA, determinar } \\
\text { resultados e elencar a classificação de } \\
\text { intervenção de enfermagem de acordo com as } \\
\text { medidas de prevenção, de abordagem inicial, de } \\
\text { cuidados e de suporte para o enfrentamento }\end{array}$ & $\begin{array}{l}\text { Estudo descritivo } \\
\text { exploratório, do tipo } \\
\text { revisão de literatura, de } \\
\text { natureza qualitativa. }\end{array}$ \\
\hline
\end{tabular}


Research, Society and Development, v. 10, n. 8, e4010816878, 2021

(CC BY 4.0) | ISSN 2525-3409 | DOI: http://dx.doi.org/10.33448/rsd-v10i8.16878

\begin{tabular}{|c|c|c|c|}
\hline & & coletivo da pandemia pela covid-19. & \\
\hline Silva et al., 2021 & $\begin{array}{lcc}\text { Aplicação do processo } & \text { de } \\
\text { enfermagem na assistência } & \text { ao } \\
\text { paciente com covid-19 } & \end{array}$ & $\begin{array}{l}\text { Identificar os possíveis diagnósticos de } \\
\text { enfermagem, embasado na taxonomia North } \\
\text { American Nursing Diagnosis Association } \\
\text { (NANDA), do paciente acometido pela covid- } \\
19 \text { e apresentar plano de cuidados para esses } \\
\text { pacientes, seguindo as taxonomias Nursing } \\
\text { Outcomes Classification (NOC) e Nursing } \\
\text { Interventions Classification (NIC) }\end{array}$ & $\begin{array}{l}\text { Revisão de literatura de } \\
\text { caráter exploratória e } \\
\text { bibliográfica }\end{array}$ \\
\hline Silva et al., 2021 & $\begin{array}{l}\text { Diagnósticos de enfermagem em } \\
\text { casos de COVID-19 com evolução } \\
\text { clínica para sepse }\end{array}$ & $\begin{array}{l}\text { Elaborar diagnósticos de enfermagem, segunda } \\
\text { a NANDA-I (2018-2020), através da } \\
\text { identificação de sinais e sintomas descritos na } \\
\text { literatura de pacientes com COVID-19 com } \\
\text { evolução clínica para sepse ou choque séptico }\end{array}$ & $\begin{array}{l}\text { Revisão narrativa da } \\
\text { literatura de cunho } \\
\text { exploratório com } \\
\text { abordagem qualitativa }\end{array}$ \\
\hline Dantas et al., 2020 & $\begin{array}{l}\text { Diagnósticos de enfermagem para } \\
\text { pacientes com covid-19 }\end{array}$ & $\begin{array}{l}\text { Elencar com base nas manifestações clínicas da } \\
\text { doença, os principais diagnósticos de } \\
\text { enfermagem que podem ser aplicados para } \\
\text { crianças, adultos, gestantes e idosos com covid- } \\
\text { 19, segundo a taxonomia da Associação Norte- } \\
\text { Americana de Diagnósticos de Enfermagem } \\
\text { (NANDA-I) versão 2018-20206, e a } \\
\text { Classificação Internacional para a Prática de } \\
\text { Enfermagem (CIPE) versão } 2019\end{array}$ & $\begin{array}{l}\text { Revisão integrativa da } \\
\text { literatura }\end{array}$ \\
\hline Barros et al., 2020 & $\begin{array}{l}\text { Contribuições da rede de pesquisa } \\
\text { em processo de enfermagem para } \\
\text { assistência na pandemia de covid- } \\
19\end{array}$ & $\begin{array}{l}\text { Descrever o processo de construção teórica dos } \\
\text { documentos de apoio ao Processo de } \\
\text { Enfermagem nos cenários de atendimento à } \\
\text { covid-19 }\end{array}$ & Relato de experiência \\
\hline Dias et al., 2021 & $\begin{array}{l}\text { Diagnósticos e intervenções de } \\
\text { enfermagem a crianças com sinais } \\
\text { respiratórios de gravidade da covid- } \\
19\end{array}$ & $\begin{array}{l}\text { Descrever afirmativas de diagnóstico e } \\
\text { intervenções de enfermagem a crianças com } \\
\text { sinais respiratórios de gravidade da covid- } 19 \text {. }\end{array}$ & $\begin{array}{l}\text { Estudo exploratório, } \\
\text { descritivo, do tipo } \\
\text { revisão narrativa }\end{array}$ \\
\hline Leite et al., 2020 & $\begin{array}{l}\text { Sistematização da assistência de } \\
\text { enfermagem aplicada ao idoso com } \\
\text { sepse }\end{array}$ & $\begin{array}{l}\text { Descrever a Sistematização da Assistência de } \\
\text { Enfermagem, respaldada na Teoria do } \\
\text { Autocuidado, a uma paciente com sepse }\end{array}$ & $\begin{array}{l}\text { Estudo qualitativo, } \\
\text { descritivo tipo estudo de } \\
\text { caso. }\end{array}$ \\
\hline Lima et al., 2021 & $\begin{array}{l}\text { Processo de enfermagem para } \\
\text { pacientes com manifestações } \\
\text { respiratórias da covid-19. }\end{array}$ & $\begin{array}{l}\text { Discutir sobre o Processo de Enfermagem } \\
\text { diante dos aspectos clínicos respiratórios da } \\
\text { covid-19. }\end{array}$ & $\begin{array}{l}\text { Estudo qualitativo, } \\
\text { descritivo, informativo } \\
\text { que partiu de uma } \\
\text { revisão narrativa da } \\
\text { literatura }\end{array}$ \\
\hline
\end{tabular}

Fonte: elaborado pelos autores (2021). 


\section{Discussão}

Após a localização dos artigos, foi possível separá-los em três categorias temáticas: processos de enfermagem, diagnósticos de enfermagem ao paciente com covid-19 e intervenções de enfermagem ao paciente com covid-19. Cada categoria será discutida individualmente, mas que permitem uma análise mais detalha

\section{Processo de enfermagem}

O covid-19 foi o responsável pelo gigantesco número de casos de infecções respiratórias nos últimos tempos. Os pacientes assintomáticos não manifestaram nenhum sinal ou sintoma da doença, o que dificultou o diagnóstico precoce e ação preventiva para evitar a disseminação do vírus. Foi possível observar que um dos principais problemas do paciente acometido pela covid-19 era o comprometimento respiratório. Entre os sintomas mais comuns, os pacientes apresentaram desde manifestações como tosse persistente até algo mais grave, que poderia ocasionar perda da função pulmonar e consequentemente, levar o paciente a óbito (Dias et al., 2021).

A prática clínica da enfermagem teve como objetivo a recuperação e prevenção da disseminação da doença, já que os pacientes acometidos pelo covid-19 após a sua recuperação, poderiam se infectar novamente e disseminar o vírus (Barros $e t$ al., 2020). A elaboração de um plano de cuidados para a equipe teve como objetivo o de proteger a equipe e o paciente de possíveis infecções cruzadas. Devido ao seu papel primordial na prevenção da infecção ocasionada pelo coronavírus, o enfermeiro deve acompanhar todo o processo de cuidado do paciente e direcionar as intervenções e cuidados. A ferramenta utilizada para nortear todo o plano de cuidado é o PE, por meio dela, juntamente com a SAE, o enfermeiro traça todo o plano terapêutico do paciente (Dantas et al., 2020).

A SAE também é utilizada para organizar as ações assistenciais do pessoal de enfermagem, assim como outras rotinas e protocolos que irão direcionar e organizar a prática da enfermagem. O PE determina o melhor caminho a ser trilhado para promover o cuidado adequado para cada paciente e por mais que todo o processo se complemente, o ponto-chave do processo que vai determinar por onde e quais ações serão implementadas, vem a partir do correto DE (Dias et al., 2021 \& Andrade et al., 2020).

Desta maneira o PE permite a identificação dos principais problemas do paciente e possibilita a atuação direta nos problemas. Os pacientes acometidos pelo covid-19 apresentam como principais diagnósticos de enfermagem a hipertermia, padrão respiratório ineficaz e trocas de gases prejudicada, na qual faz necessário a atuação direta em promover o conforto respiratório do paciente e garantir a normalização dos sinais vitais (Leite et al., 2020 \& Lima et al., 2021). Esses diagnósticos são discutidos com mais detalhes na próxima categoria temática.

\section{Principais diagnósticos de enfermagem}

O DE faz parte do PE e é um dos métodos que irá determinar quais tipos de ações e intervenções serão aplicados na assistência para que os resultados esperados venham ser alcançados. Ao elaborar diagnósticos de enfermagem para paciente com covid-19 é necessário considerar os aspectos biopsicossociais, para que as intervenções atendam às suas necessidades. Estudos mostram que entre os sinais e sintomas causados pelo covid-19, os mais frequentes são tosse, dispneia e hipertermia e dessa forma, ao aplicar os diagnósticos de enfermagem, o enfermeiro deve considerar esses sintomas (Barros et al., 2020; Dantas et al., 2020; Leite et al., 2020 \& Lima et al., 2021).

O julgamento clínico das respostas do indivíduo é o que vai nortear o correto DE e isso é uma parte essencial para traçar o plano de cuidados do paciente no processo saúde-doença. Dessa forma, os DE encontrados com maior frequência em pacientes acometidos pelo vírus do covid-19 estão associados ao sistema respiratório. Os diagnósticos voltados para o padrão ventilatório do paciente são a respiração ineficaz e troca de gases prejudicado, mas os pacientes podem apresentar diagnósticos 
de risco de infecção e outros relacionados ao sistema gastrointestinal, como diarreia, além de afetar os mecanismos de regulação que poderá ocasionar a hipertermia (Barros et al., 2020 \& Dantas et al., 2020). Ainda com relação aos diagnósticos de enfermagem, estes são apresentados, de acordo com a NANDA-I e a classificação internacional da prática de enfermagem (CIPE):

Domínio 1. Promoção da saúde: proteção ineficaz relacionado à incapacidade de proteção contra agente infeccioso evidenciado por tosse, calafrios e fadiga.

Domínio 2. Nutrição: risco de desequilíbrio eletrolítico relacionado a presença de vômito e diarreia, volume de líquidos excessivo relacionado a retenção hídrica evidenciada por edema em membros inferiores.

Domínio 3. Eliminação e troca: diarreia relacionada a processo infeccioso evidenciada por dor abdominal, troca de gases prejudicada relacionada ao comprometimento pulmonar evidenciado por dispneia e padrão respiratório anormal.

Domínio 4. Atividade/repouso: débito cardíaco diminuído relacionado a processo patológico evidenciado por palpitações cardíacas e dispneia, fadiga relacionada a processo patológico evidenciada por aumento dos sintomas físicos, padrão respiratório ineficaz relacionado a dor e fadiga evidenciado por dispneia, ventilação espontânea prejudicada relacionado a fadiga da musculatura respiratória por evidenciada por dispneia.

Domínio 10. Princípios de vida: sofrimento espiritual relacionado a depressão, estressores e mudança ambiental evidenciado por insônia.

Domínio 11. Segurança/proteção: hipertermia relacionada a processo infeccioso evidenciado por pele quente ao toque no paciente.

Domínio 12. Conforto: dor aguda relacionada a agente biológica lesiva evidenciada por relato de comportamento de dor/alterações nas atividades, náusea relacionada a exposição a toxinas evidenciada por ânsia de vômito.

Já os DE da CIPE ${ }^{\circledR}$ foram: dor muscular, dor musculoesquelética, dor no peito, presença de corrimento nasal, presença de dispneia, presença de edema em pernas, presença de febre, presença de hipertermia, presença de tosse e presença de vômito e diarreia.

Andrade et al., (2020) descreve a tosse como uma das manifestações clínicas mais frequentes e que pode ser encontrada nas características definidoras dos seguintes DE: "deglutição prejudicada", "nutrição desequilibrada: menor do que as necessidades corporais", "desobstrução ineficaz das vias aéreas", "insônia", "conforto prejudicado" e "dor aguda". A febre também é um sintoma amplamente relatado, e para essa manifestação clínica deve-se utilizar o diagnóstico de enfermagem "hipertermia".

Alguns pacientes apresentam mialgia, nesse caso os seguintes diagnósticos de enfermagem podem ser utilizados: "mobilidade física prejudicada", "fadiga", "intolerância à atividade", "conforto prejudicado" e "dor aguda". No momento em que o paciente apresenta dispneia, pode utilizar os seguintes diagnósticos de enfermagem: "insônia", "padrão respiratório ineficaz", "ventilação espontânea prejudicada", "desobstrução ineficaz das vias aéreas" e "conforto prejudicado". A dor de garganta é um sintoma pouco frequente. Três diagnósticos de enfermagem foram identificados para essa manifestação clínica: "deglutição prejudicada", "nutrição desequilibrada: menor que as necessidades corporais" e "dor aguda".

Os DE mais utilizados para a fadiga são: "mobilidade física prejudicada", "fadiga", "intolerância à atividade" e "conforto prejudicado". Na presença de coriza, pode-se utilizar os seguintes diagnósticos: "risco de integridade tissular prejudicada" e "conforto prejudicado" (Andrade et al., 2020). Outros diagnósticos importantes encontrados nos pacientes com covid-19 estão relacionados ao sistema emocional que pode desenvolver altos níveis de ansiedade, com mudanças no padrão comportamental e psíquico. Dessa forma, o enfermeiro precisa se atentar e identificar os principais problemas apresentados pelo paciente, a ponto de sugerir e implantar os cuidados e intervenções ideais para esses pacientes (Barros et al., 2020 \& Dantas et al., 2020). 
Portanto, o conhecimento adequado do tipo de cuidado que será implementado é primordial para que o haja um controle do desenvolvimento da doença. O DE ideal é aquele baseado nos sinais e sintomas e claro, no desenvolvimento da doença, o que garante a implementação assertiva dos cuidados oferecidos pela equipe de enfermagem, diminui os riscos de acometimento ocasionado pela covid-19 (Leite et al., 2020 \& Lima et al., 2021).

Em relação às crianças, de acordo com Dias et al. (2021) os principais sinais de gravidade do paciente com covid-19 são: "dispneia" e "desconforto respiratório". Nesse sentido, utilizou-se a taxonomia da NANDA-I, com foco na Classe 4: Respostas Cardiovasculares/Pulmonares. Os DE pertencem a duas classes que são o domínio 3 - eliminação/troca, diagnóstico: troca de gases prejudicada e domínio 4 - atividade/repouso, diagnósticos: padrão respiratório ineficaz, ventilação espontânea prejudicada.

Ainda segundo Dias, et al. (2020) em crianças e adolescentes, as alterações mais evidentes são nos sistemas cardiovascular, gastrointestinal e tegumentar. Os seguintes sinais e sintomas destacam-se: dor abdominal, vômito, diarreia, taquicardia, disfunção ventricular, febre, erupção cutânea e hiperemia conjuntival. Dessa forma os seguintes DE foram elencados a partir dos indicadores frequentemente encontrados: "proteção ineficaz", "nutrição desequilibrada: menor que as necessidades corporais", "diarreia", "motilidade gastrintestinal disfuncional", "troca de gases prejudicada", "padrão respiratório ineficaz", "deambulação prejudicada", "risco de perfusão tissular cerebral ineficaz", "risco de confusão aguda", "confusão aguda", "integridade da membrana mucosa oral prejudicada", "integridade da pele prejudicada", "hipertermia", "risco de choque", "risco de lesão na córnea", "risco de tromboembolismo venoso", "risco de quedas" e "dor aguda" (Dias et al., 2020).

Os principais DE para o paciente que será submetido a cirurgia são: medo, ansiedade, déficit de conhecimento no préoperatório, dor aguda, padrão respiratório ineficaz, intolerância à atividade, risco de infecção, risco de desequilíbrio no volume de líquidos, risco de aspiração e risco de desequilíbrio na temperatura corporal. Nos estudos de Menezes et al. (2020) também foram descritos os seguintes diagnósticos:

1. Padrão respiratório ineficaz, evidenciado por: padrão respiratório anormal. Relacionado à: fadiga

2. Troca de gases prejudicada, evidenciada por: padrão respiratório anormal. Relacionada a: desequilíbrio na relação ventilação-perfusão.

3. Síndrome do idoso frágil, evidenciada por: débito cardíaco diminuído. Relacionado à: imobilidade e intolerância à atividade.

4. Débito cardíaco diminuído, evidenciado por: taquicardia e oligúria. Relacionado à: alteração na frequência cardíaca.

5. Volume de líquidos excessivos, evidenciado por: edema, ruído respiratório, adventício (sibilo) e/ou oligúria.

6. Relacionado a: mecanismo regulador comprometido

7. Dor aguda, evidenciada por: comportamento expressivo; expressão facial de dor, mudanças nos parâmetros fisiológicos normais, gestos de proteção, relacionada a agente biológico lesivo.

8. Integridade tissular prejudicada, evidenciada por: tecido lesado, relacionada a: fator mecânico, mobilidade prejudicada, neuropatia periférica.

\section{Principais intervenções de enfermagem}

Os DE apresentados na categoria anterior requerem que os sinais vitais, padrão respiratório e nível de saturação de oxigênio sejam monitorados. Em situações nas quais ocorre a indução anestésica existe o risco de aspiração, pois o paciente perde os reflexos laríngeos e isso pode agravar mais ainda algum problema respiratório. Após a sedação, entre as principais intervenções de enfermagem estão o controle dos sinais vitais, balanço hídrico, monitoramento contínuo de saturação, pressão arterial, temperatura, sinais de hipóxia, esforço respiratório ou cianose. Nesse contexto, os cuidados na anestesia de pacientes 
com covid-19 incluem a oferta de oxigênio por meio de máscara com reservatório, aspiração através de sistema fechado, utilizar o filtro HEPA (High Efficiency Particulate Arrestance) no ramo expiratório e no Y acoplado na máscara do circuito respiratório (Cesário et al., 2021; Melo et al., 2021; Menezes et al., 2020 \& Moura et al., 2020). Os cuidados típicos da rotina de uma unidade intensiva também se aplicam nestes pacientes.

O diagnostico irá determinar qual o tipo de problema e, a partir daí, qual os necessários cuidados têm que ser executados para que o paciente se recupere. A avaliação constante do diagnóstico implantado irá determinar se ele ainda responde à necessidade do paciente ou não. A enfermagem elabora um importante papel no processo de recuperação do paciente, pois é a responsável por acompanhar o paciente durante todo esse processo. Cabe ao enfermeiro gerenciar todo o cuidado direcionado a esse paciente e analisar se há a necessidade de mudanças de ações durante o cuidado (Dias et al., 2021 \& Melo et al., 2021).

Ao diagnosticar um paciente com sepse, por exemplo, é de extrema relevância identificar qual o tipo de sepse e como se originou, pois assim o processo de cuidado será preciso, com foco na resolução do problema, bem-estar e consequente recuperação do paciente. Na assistência crítica a enfermagem precisa organizar e sistematizar o cuidado, nesse sentido as terminologias permitem a identificação e documentação de padrões de cuidados. Por meio da CIPE os enfermeiros podem coletar, descrever e documentar sistematicamente os elementos da prática de enfermagem (Menezes et al., 2020).

Neste sentido, um estudo brasileiro aponta que a hospitalização por síndrome respiratória aguda grave desde a detecção do primeiro caso de covid-19 no brasil superou o observado e que a hospitalização de casos graves de covid-19 já consiste em uma sobrecarga para o sistema de saúde. A emergência é a porta de entrada dos hospitais e nos casos de covid-19 e é onde o enfermeiro aplica critérios de classificação de risco para adequada identificação e atendimento dos casos, visto que a avaliação clínica e a tomada de decisão do enfermeiro são importantes para priorizar os pacientes que mais necessitam de atendimento médico (Queiroz et al., 2020; Ramos et al., 2020; Silva et al., 2021 \& Silva et al., 2021).

Portanto, reconhece-se a equipe de enfermagem como protagonista no combate à transmissão da doença, visto que é uma categoria profissional numerosa e acompanha o paciente durante as 24 horas. Esse protagonismo já foi descrito em estudo realizado por Cesário, Flauzino e Mejia (2020), que descreveu a atuação da enfermagem no combate ao covid-19.

\section{Considerações finais}

A aplicação correta do PE e a identificação adequada do DE contribuem para a resolução do problema identificado, pois permite as intervenções mais apropriadas, baseadas nas reais necessidades do paciente. A SAE e o PE permitem a padronização do atendimento e funcionamento da equipe de enfermagem, a avaliação adequada das necessidades e priorização dos problemas, assim como permite ao enfermeiro delegar as atividades corretas para sua equipe. Devido à complexidade dos cuidados com o paciente com covid-19, principalmente nas unidades de emergência e de terapia intensiva, é importante que os profissionais de enfermagem tenham um olhar crítico e direcionado para que todos os cuidados sejam administrados de forma efetiva.

Foi possível identificar 12 diagnósticos frequentes em pacientes com covid-19, que são: promoção da saúde: proteção ineficaz, risco de desequilíbrio eletrolítico, diarreia, débito cardíaco diminuído, sofrimento espiritual, hipertermia relacionada, dor aguda relacionada. Um diagnóstico que foi encontrado com frequência na literatura é o de diarreia, que além de afetar os mecanismos de regulação que poderá ocasionar a hipertermia que irá ocasionar um grande desequilíbrio hidroeletrolítico. No entanto muitas vezes na prática, é um DE pouco utilizado, por não possuir uma relação direta com o sistema respiratório. Para a adequada confirmação dos $\mathrm{DE}$ encontrados nesta revisão, faz-se necessária uma pesquisa in locu, tanto em unidades de pronto atendimento, quanto em unidades de terapia intensiva que atendam casos confirmados de covid-19. 
Por fim, constatou-se uma escassez de publicações nacionais a respeito do tema abordado nesta pesquisa, com isto os autores esperam que este estudo contribua para o desenvolvimento de novas pesquisas relacionadas aos diagnósticos de enfermagem em pacientes acometidos pelo covid-19. Quando bem aplicado, o DE impacta de maneira positiva na assistência de enfermagem e contribuir para uma melhor recuperação do paciente, além de evitar intervenções desnecessárias.

\section{Referências}

Andrade, T. R. S. F., et al. (2021). Assistência de enfermagem aos casos leves da COVID-19. Research, Society and Development. 10(1): e5310111307. $10.33448 /$ rsd-v10i1.11307.

Andrade, T. R. S. F., et al. (2020). Principais diagnósticos de enfermagem em pacientes com manifestações clínicas da covid-19. Revista Eletrônica Acervo Saúde. 12(10) e4883. https://doi.org/10.25248/reas.e4883.2020.

Barros, A. L. B. L., et al. (2020). Contribuições da rede de pesquisa em processo de enfermagem para assistência na pandemia de COVID-19. Revista Brasileira Enfermagem. 73(2): e20200798. https://doi.org/10.1590/0034-7167-2020-0798.

Cardoso, R. S. S., et al. (2020). Diagnóstico, resultados e intervenções de enfermagem ao idoso frágil, acometido por covid-19: uma análise documental. Research, Society and Development. 9(9): e239997109. 10.33448/rsd-v9i9.7109.

Cesário, J. M. S., et al. (2020). O protagonismo da enfermagem no combate do COVID-19. Revista Científica Multidisciplinar Núcleo do Conhecimento. 11(25). 149-168. 10.32749/nucleodoconhecimento.com.br/saude/protagonismo-da-enfermagem

Cesário, J. M. S., et al. (2021a). Cuidados de enfermagem para o paciente com infarto agudo do miocárdio portador de covid - 19. Revista Científica Multidisciplinar Núcleo do Conhecimento. 12(02). 61-76. 10.32749/nucleodoconhecimento.com.br/saude/infarto-agudo.

Cesário, J. M. S., et al. (2021b). Cuidados de enfermagem durante a anestesia de paciente com COVID-19. Revista Científica Multidisciplinar Núcleo do Conhecimento. 02(06). 103-116. 10.32749/nucleodoconhecimento.com.br/saude/anestesia-de-paciente.

Cesário, J. M. S., et al. (2021c). Cuidados de enfermagem durante a anestesia de paciente com covid-19. Revista Científica Multidisciplinar Núcleo do Conhecimento. 6(2).103-116. 10.32749/nucleodoconhecimento.com.br/saude/anestesia-de-paciente.

Cesário, J. M. S., Flauzino, V. H. P., \& Mejia, J. V. C. Metodologia científica: Principais tipos de pesquisas e suas caraterísticas. Revista Científica Multidisciplinar Núcleo do Conhecimento. 5(11). 23-33. 10.32749/nucleodoconhecimento.com.br/educacao/tipos-de-pesquisas.

Dantas, T. P., et al. (2020). Diagnósticos de Enfermagem para pacientes com COVID-19. Journal Health NPEPS. 5(1). 396-416. 10.30681/252610104575.

Dias, R. B. F., et al. (2020). Diagnósticos de enfermagem para pacientes com síndrome inflamatória multissistêmica pediátrica associada à covid-19: revisão integrativa. Revista Norte Mineira de enfermagem. 9(2).46-56. 10.46551/rnm23173092202090205.

Dias, R. B. F., et al. (2021). Diagnósticos e intervenções de enfermagem a crianças com sinais respiratórios de gravidade da covid-19. Revista Baiana de Enfermagem. 35(1): e37467. http://dx.doi.org/10.18471/rbe.v35.37467.

Gallaschi, C. H., et al. (2020). Prevenção relacionada à exposição ocupacional do profissional de saúde no cenário de covid-19. Revista de enfermagem da UERJ. 1(28): e49596. http://dx.doi.org/10.12957/reuerj.2020.49596.

Humerez, D. C., Ohl, R. I. B., \& Silva, M. C. N. (2020). Saúde mental dos profissionais de enfermagem do Brasil no contexto da pandemia Covid-19: ação do Conselho Federal de Enfermagem. Cogitare enfermagem. 1(25): e74115. http://dx.doi.org/10.5380/ce.v25i0.74115.

John Hopkins Coronavirus Resource Center. (n.d.). United States cases by county. Johns Hopkins University \& Medicine. https://coronavirus.jhu.edu/us-map.

Leite, F. C. S., et al. (2020). Sistematização da assistência de enfermagem aplicada ao idoso com sepse. Revista de Enfermagem UFPE, v. 13(1). 1-9. $10.5205 / 1981-8963.2020 .244715$.

Lima, L. S., et al. (2020). Processo de enfermagem para pacientes com manifestações respiratórias da covid-19. Revista de Enfermagem UFPE. 15(1). 1-10 .10.18471/rbe.v35.37467.

Medeiros, E. A. S. (2020). A luta dos profissionais de saúde no enfrentamento da covid-19. Acta Paul Enfermagem. 33: e-EDT20200003. http://dx.doi.org/10.37689/acta-ape/2020EDT0003.

Melo, E. C. A., et al. (2021). Processo de enfermagem aplicado ao paciente com COVID-19 a partir de uma scoping review. Research, Society and Development. 10(2): e53810212741. 10.33448/rsd-v10i2.12741.

Menezes, H. F., et al. (2020). Terminologia especializada de enfermagem para a prática clínica à COVID-19. Texto contexto enfermagem. 1(29): e20200171. https://doi.org/10.1590/1980-265x-tce-2020-0171.

Moura, A. V. S. M., et al. (2020) "NANDA COVID-19": relato da construção de panfleto educativo sobre os diagnósticos de enfermagem às pessoas infectadas com o SARS-CoV-2. Conexão Unifametro 2020 XVI Semana Acadêmica.

Nascimento, T. F., et al. (2021). Infecções por coronavírus: planejamento da assistência fundamentado na Teoria de Enfermagem de Orem. Revista Brasileira de Enfermagem. 74(1): e20200281. 10.1590/0034-7167-2020-0281. 
Research, Society and Development, v. 10, n. 8, e4010816878, 2021

(CC BY 4.0) | ISSN 2525-3409 | DOI: http://dx.doi.org/10.33448/rsd-v10i8.16878

Peters, M., et al. (2015) Guidance for conducting systematic scoping reviews. International Journal of Evidence-Based Healthcare. 13(3). 141-146. 10.1097/XEB.0000000000000050.

Queiroz, A. G. S., et al. (2020). Diagnósticos de enfermagem segundo a taxonomia da NANDA internacional para sistematização da assistência de enfermagem a covid-19. Journal of Health \& Biological Sciences. 8(1).1-6. 10.12662/2317-3206jhbs.v8i1.3352. 2020.

Ramos, C. R., et al. (2020). Diagnósticos, resultados e intervenções de enfermagem voltados para comunidade, na pandemia de covid-19. Research, Society and Development. 9(9): e933997400. 10.33448/rsd-v9i9.7400.

Silva, A. L. O., et al. (2021). Aplicação do processo de enfermagem no cuidado ao paciente com COVID-19. Research, Society and Development. 10(3). e6610313056. 10.33448/rsd-v10i3.13056.

Silva, M. I. C., et al. (2021). Diagnósticos de enfermagem em casos de covid-19 com evolução clínica para sepse. Research, Society and Development. 10(1): e17410111232. 10.33448/rsd-v10i1.11232.

Silva, J. M. A. V., et al. (2020). Planejamento organizacional no contexto de pandemia por covid-19: implicações para a gestão em enfermagem. Journal Health NPEPS. 5 (1):e4626. http://dx.doi.org/10.30681/252610104626.

Sousa, A. R., et al. (2020). Reflexões sobre o Processo de Enfermagem no trabalho de enfermeiras frente à pandemia da covid-19. Revista Enfermagem em Foco. 11(1). 62-67. 